Journal for

ImmunoTherapy of Cancer

\title{
Complement controls the immune synapse and tumors control complement
}

\author{
Alan Herbert
}

To cite: Herbert A. Complement controls the immune synapse and tumors control complement. Journal for ImmunoTherapy of Cancer 2020;8:e001712. doi:10.1136/jitc-2020-001712

Accepted 31 October 2020

\section{Check for updates}

(C) Author(s) (or their employer(s)) 2020. Re-use permitted under CC BY-NC. No commercial re-use. See rights and permissions. Published by BMJ.

Discovery, InsideOutBio Inc, Charlestown, Massachusetts, USA

Correspondence to

Dr Alan Herbert;

alan.herbert@insideoutbio.com

\section{ABSTRACT}

The synapses between immune cells and their targets are $150 \AA ̊$ wide. They regulate immune cell responses (IRs) to cognate antigens. Here, I outline a potential mechanism for self-nonself discrimination based on the C3d and iC3b proteolytic fragments of complement protein C3. The proposed C3 checkpoint works through complement receptor 3 (CR3), which binds both C3d and iC3b. The CR3 conformations involved differ; the bent, cis-acting CR3 engages C3d, activating the immune cell expressing CR3; the extended, transacting CR3 conformer binds iC3b on another cell, inhibiting IRs. The CR3 complexes formed with iC3b and C3d vary greatly in size. Only bound C3d is small enough to fit within the synapse. It stimulates IRs by countering the inhibitory signals that iC3b generates at the synapse edge. The competition between C3d and iC3b dynamically determines whether or not an immune cell activates. Host cells use regulators of complement activation (RCA) to coat themselves with iC3b, silencing IRs against self by preventing synapse formation. Tumors exploit this process by overexpressing C3 and RCA to masquerade as 'super-self', with iC3b masking neoantigens. Enhancing synapse formation by specifically labeling cancer cells as nonself with targeted C3d therapeutics offers a new strategy for boosting tumorspecific immunity.

\section{THE PARADOX OF C3}

The complement system is an ancient mechanism for self-nonself discrimination. Its role in cancer is paradoxical. ${ }^{1}$ Both complement activation and complement deficiency can promote tumor growth. For example, in genetic and syngeneic models of epithelial ovarian carcinoma, deletion of the complement C3 gene inhibits tumor growth. Similarly, in mouse models of melanoma, complement C3 deficiency delays progression. ${ }^{1}$ In contrast, C3 deficiency in the Her2/ neu autochthonous mouse mammary carcinoma model leads to an earlier onset and accelerated tumor spread. In this opinion piece, I explore the complement paradox and integrate existing data to advance its resolution. I will describe a complement C3 checkpoint used by tumors to label themselves as 'super-self'. By doing so, cancer cells silence immune responses (IRs) directed at neoantigens. In this scenario, iC3b leads to tolerance by labeling cells as 'self' while C3d initiates immune activation by signaling "nonself' (figure 1).

\section{COMPLEMENT BASICS}

Complement was discovered by Buchner and Bordet in the 1890s and has since been intensely studied by many distinguished scientists to give a detailed view of the proteins and the molecular interactions involved. The C3 protein plays a key role in the complement cascade. ${ }^{23}$ Once activated to form C3b, a positive feedback loop accelerates the conversion of additional C3 to C3b (figure 1A). The cycle is broken by the regulators of complement activity (RCA). RCAs act on C3b to coat host cells with iC3b, producing non-inflammatory outcomes. Pathogens lacking RCAs are tagged instead with the proteolytic fragment C3d, which promotes immunity. ${ }^{4}$

\section{THE TAG TEAM}

The biology of iC3b and C3d is fundamentally different. The receptors they engage and their size likely determines the outcomes they produce (figure 1C,D.). Size matters. At $\sim 45 \AA$ (PDB: 4M76), C3d is likely small enough to fit within the immune synapse (IS), which is $150 \AA$ wide (figure 2). ${ }^{5}$ There it potentially modulates the signals that induce immune cell (IC) responses. In contrast, $\mathrm{iC} 3 \mathrm{~b}$ with a maximal dimension of $\sim 183 \AA^{6}$ is likely too large to fit within the IS.

\section{THE MANY FACES OF COMPLEMENT RECEPTOR 3}

C3d and iC3b interact with the complement receptor 3 (CR3, Mac-1, CD18/CD11b, $\alpha \mathrm{M}$ / $\beta 2$ encoded by ITGAM/ITG2B2 respectively), but they bind different CR3 conformations. ${ }^{7}$ CR3 traditionally is considered to adopt three states: extended and high affinity $(\mathrm{E}+\mathrm{H}+)$, bent and low affinity (E-H-), extended and low affinity (E+H-). ${ }^{7}$ Recent electronmicroscopy evidence supports a fourth CR3 state that is bent and of high affinity $(\mathrm{E}-\mathrm{H}+)$ 


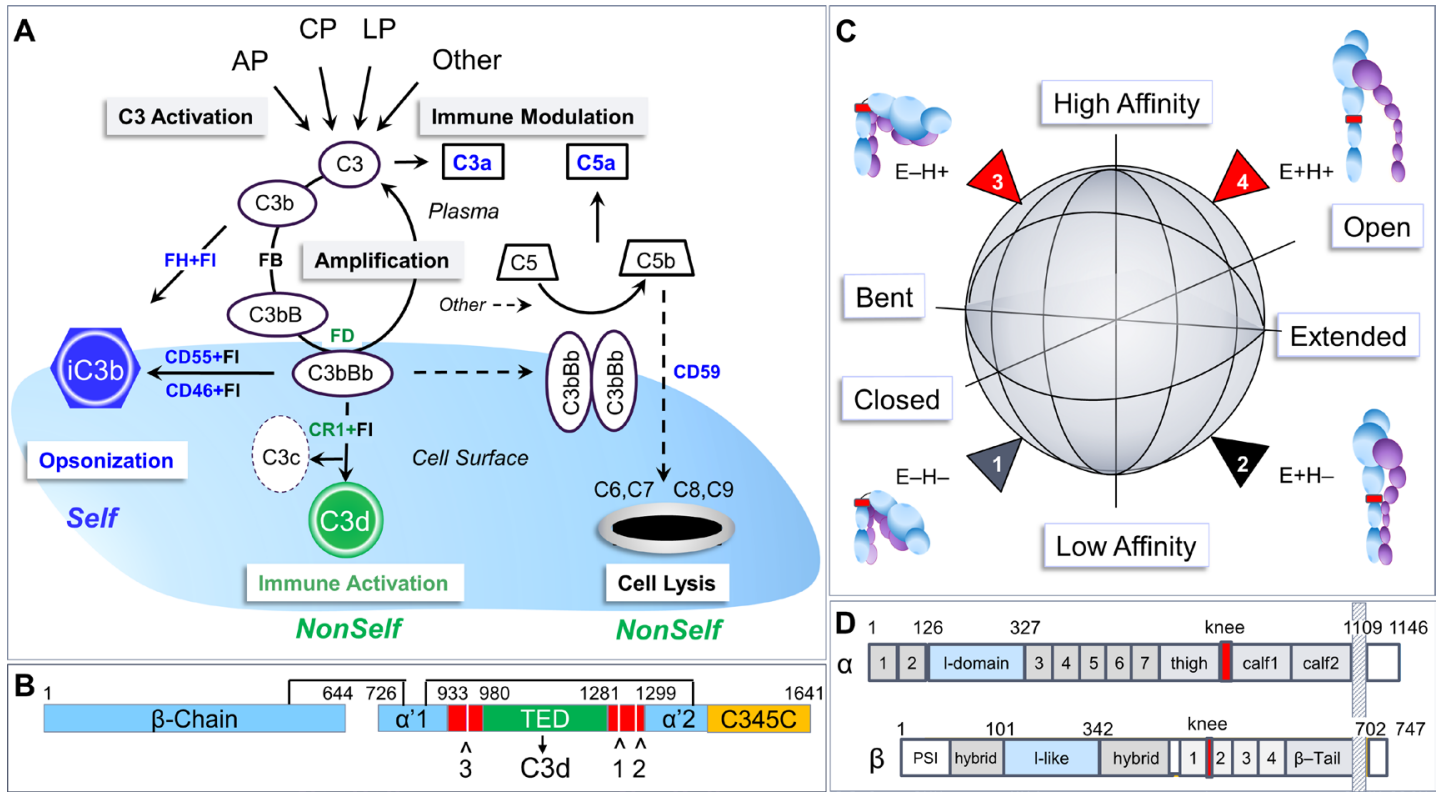

Figure 1 The role of complement C3 in self-nonself discrimination. (A) The alternative (AP), classical (CP), lectin (LP) and other ${ }^{16}$ pathways drive the complement amplification loop driven by complement factors $B$ and $D$ (FB, FD) to produce the $\mathrm{C} 3 \mathrm{~b}$ convertase that activates additional $\mathrm{C} 3$ and $\mathrm{C} 5$ proteins. C3b is inactivated by compactor factor I (FI) to form either iC3b or C3d fragments. Complement factor $\mathrm{H}(\mathrm{FH})$, CD46 and CD55 favor iC3b production while Complement Receptor 1 (CR1) favors C3d formation by releasing the large C3c fragment. Tumors use the iC3b fragment to label themselves as 'super-self' to silence immune responses against the abnormal proteins they produce. CD59 prevents complement-mediated lysis of tumors by Complement $\mathrm{C} 6, \mathrm{C} 7, \mathrm{C} 8$ and $\mathrm{C} 9$ that together form a membrane pore. It is proposed here that $\mathrm{C} 3 \mathrm{~d}$ tags cells as nonself and favors antitumor responses. (B) Linear representation of C3b domains with cleavage sites indicated by ^ and a white line. The order of cleavage is indicated by the numbers. Cleavages 1 and 2 produce iC3b by releasing C3f. The third cleavage at the other end of the thioester domain (TED) results in the production of C3dg which is trimmed by tissue proteases to give C3d. Cleavage releases $\mathrm{C} 3 \mathrm{c}$ which consists of the $\beta$-chain connected to the $\alpha^{\prime} 1$ and $\alpha^{\prime} 2$ domains by disulfide bonds (indicated by lines above the domains). The C345C domain combines with C3d to create the CR3 binding site. (C) The four conformations of complement receptor $3(\mathrm{CR} 3)$ with active states colored red. The bent (E-) or extended ( $\mathrm{E}+$ ) conformations and the low $(\mathrm{H}-)$ or high affinity $(\mathrm{H}+)$ affinity states of each play different roles in immune regulation as described in the text. The open state is associated with outside-in signaling. CR3 can transition from the bent inactive state to the fully extended high affinity state by path $1 \rightarrow 2 \rightarrow$ or $1 \rightarrow 3 \rightarrow 4 .^{7}$ The E- $\mathrm{H}+$ state is visualized in the crystal structure of the $\alpha \times \beta 2$ integrin ectodomain. ${ }^{17}$ (D) The domains of the $\mathrm{CR} 3 \alpha(\mathrm{CD} 11 \mathrm{~b})$ and $\beta$ (CD18) chains with the I domain and I-like domain shown in blue and the flexible knee that bends in red.

(figure 1C) that binds extracellular ligands through the $\alpha \mathrm{M}$ I-domain (figure 1D). ${ }^{8}$ The bent state projects only $110 \AA$ from the cell surface (figure 2D) and is small enough to fit within an IS. FRET studies of neutrophils reveal that bent CR3(E-H+) binds the intercellular adhesion molecule 1 (ICAM1) on the same cell (ie, in cis) and inhibits binding of extended $\mathrm{CR} 3(\mathrm{E}+\mathrm{H}+)$ to ICAM1 on another surface (ie, in trans). ${ }^{7}$ Super-resolution microscopy further enables quantitation of the how the four CR3 states cluster together and how these interactions affect cellular responses. ${ }^{7}$

The bent CR3 (E-H+) can only bind complement fragments through its $\alpha \mathrm{M}$ I-domain as other binding surfaces are buried (figure 2D). ${ }^{6}$ The $\alpha \mathrm{M}$ I-domain binds C3d with high nanomolar affinity (KD $\sim 0.4 \mu \mathrm{M}){ }^{2}$ The lower overall affinity of bent CR3(E-H+) for iC3b suggests that its C3d domain is often occluded and not available for binding. ${ }^{26}$

The interaction of bent CR3(E-H+) with C3d likely occurs in cis (ie, on the same cell surface that CR3 is on) (figure 2B), similar to the interactions of bent CR3(E-H+) with ICAM1. ${ }^{7}$ The complex formed is likely small enough to fit within an
IS (figure 2A,B). The affinity of extended $\mathrm{CR} 3(\mathrm{E}+\mathrm{H}+$ ) for iC3b in trans is high due to binding sites newly exposed that engage iC3b by bringing its C3d and C345C domains together (figure 2D). ${ }^{6}$ By analogy with the neutrophil findings, the binding of bent $\mathrm{CR} 3(\mathrm{E}-\mathrm{H}+)$ to $\mathrm{C} 3 \mathrm{~d}$ in cis likely competes with the interactions of extended CR3(E+H+) with iC3b in trans. Whether cis or trans outcomes predominate depends on the density of C3d and iC3b on each cell partner. The outcomes are binary as each ligand inhibits the binding of CR3 to the other.

\section{GOING BIG}

The CR3 $(\mathrm{E}+\mathrm{H}+)$ extended conformation is the default conformation on macrophages. ${ }^{9}$ The interaction with iC3b inhibits T-Cell activation independently of any process related to antigen presentation. ${ }^{9}$ The clusters formed promote phagocytosis. ${ }^{10}$ In the case of tumors, the outcome is non-inflammatory. ${ }^{4}$

Antibody-mediated phagocytosis is more complex. It involves both extended CR3 $(\mathrm{E}+\mathrm{H}+)$ and bent $\mathrm{CR} 3(\mathrm{E}-\mathrm{H}+)$. 


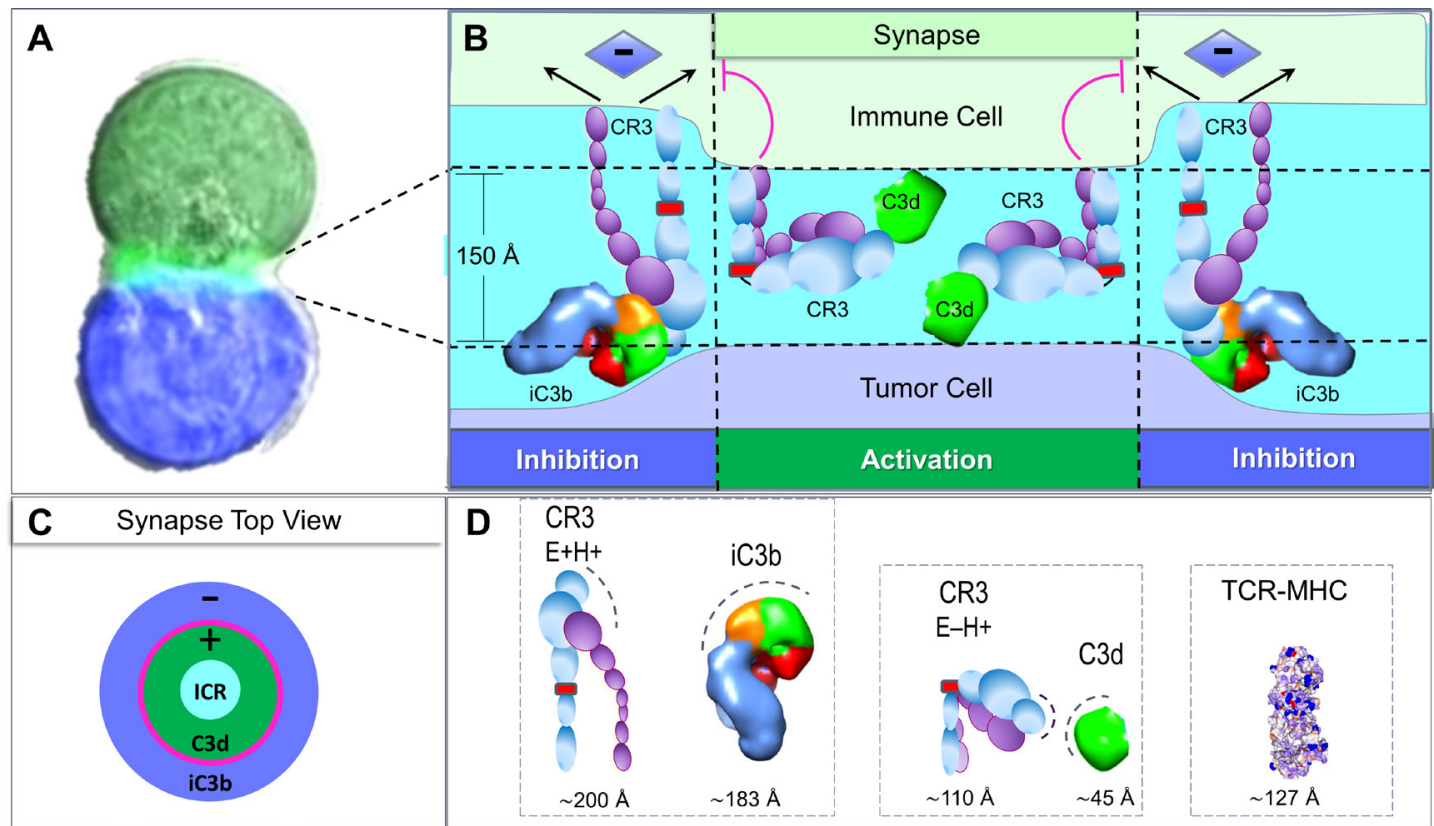

Figure 2 Complement and the immune synapse. It is proposed that complement dependent immune outcomes depend on latent binding sites on both CR3 and C3 as well as the size of the C3 proteolytic Fragments. (A) An immunological synapse formed between two cells is shown in light green (source https://en.wikipedia.org/wiki/lmmunological_synapse). (B) The complex of iC3b with the high affinity, extended form of $\mathrm{CR} 3(\mathrm{E}+\mathrm{H}+)$ (see legend figure 1) is likely too large to fit within the synapse width. Instead, the complex acts on the cytoskeleton to oppose synapse formation and inhibit immune cell activation. The small size of $\mathrm{C} 3 \mathrm{~d}$ and the bent $\mathrm{CR} 3(\mathrm{E}-\mathrm{H}+)$ receptor likely allows their accommodation within a synapse. The activated bent $\mathrm{CR} 3(\mathrm{E}-\mathrm{H}+)$ opposes the cytoskeletal forces generated by iC3b that would otherwise collapse the synapse. Instead those forces, now unbalanced, promote synapse expansion by pulling outwards on the synapse edge. (C) A top overview of the synapse illustrating the different zones of activation and inhibition surrounding the central zone of antigen recognition by the immune cell receptor (ICR). The magenta line indicates the ring of competition between bent $\mathrm{CR} 3(\mathrm{E}-\mathrm{H}+)$ and extended CR3 $(\mathrm{E}+\mathrm{H}+$ ). (D) The size of iC3b (PDB: 2A73) and C3d (PDB: 4M76) fragments are compared with those of the extended and bent form of CR3 with latent binding sites indicated by a dashed line (adapted from ${ }^{6}$ ). The knee at which CR3 flexes is shown in red. The C3d domain is colored green and the C345C domain orange as in figure 1. The size of the T-cell receptor, major histocompatibility complex, peptide is shown for comparison (PDB: 3RGV). CR3, complement receptor 3; E+H+, extended and high affinity.

Here antibodies bridge small antigens (less than $150 \AA$ ) on one cell to a Fc receptor (FcR) on the other (figure $2 \mathrm{E}) .{ }^{10}$ The FcR interacts with bent CR3(E-H+) in cis to form a synapse that leads to phagocyte activation. The structure formed is encircled by extended CR3 $(\mathrm{E}+\mathrm{H}+) / \mathrm{iC} 3 \mathrm{~b}$ complexes that signal in trans, excluding inhibitory phosphatases like CD45 and CD148 from the synapse while engaging the cytoskeleton to initiate phagocytosis. ${ }^{9} 10$ The process is dynamic, with competition between bent and extended CR3 determining the threshold for phagocytosis. $^{7910}$

\section{STAYING SMALL}

In contrast to the inhibition of T-Cells by fully extended CR3 $(\mathrm{E}+\mathrm{H}+)$, bent CR3(E-H+) can activate ICs. CR3 is expressed on T-Cells following antigen exposure and during primary and secondary antiviral responses in mice. Mice with CR3 deficiency show impaired T-Cell responses to the T-Cell super antigen staphylococcal enterotoxin A. ${ }^{9}$ The defect is in the T-cells themselves, not in the myeloid cells they bind. Stimulation of allogeneic IRs also depends on T-Cell expression of CR3. ${ }^{9}$ Human T-cells coated with covalently bound C3d show enhanced cytokine secretion after stimulation compared with C3d negative cells, both in patients with systemic lupus erythematosus and in normal subjects. ${ }^{11}$ Unmodified C3d expressed in tumors also improves their effectiveness as anti-tumor vaccines and enhances checkpoint inhibitor efficacy. ${ }^{12}$ Collectively these results are consistent with the notion that engagement of bent CR3(E-H+) with $\mathrm{C} 3 \mathrm{~d}$ activates ICs. This cis signaling event potentially involves C3d on both sides of the synapse as the two cell membranes are closely approximated (figure 2B). In this scenario, C3d initiates immune activation by signaling 'non-self' while iC3b produces tolerance by tagging cells as 'self'.

\section{TUMORS OWN THE IR}

The competition between trans-inhibition and cisstimulation of cell-mediated immunity offers new mechanistic insight into how tumors might evade IRs. Overproduction by tumors of both C3 (see https://www. proteinatlas.org/ENSG00000125730-C3/pathology for immunohistopathology performed with different antibodies) and RCA favors iC3b formation and the extended 
$\mathrm{CR} 3(\mathrm{E}+\mathrm{H}+)$ interactions that lead to immune suppression. In the scenario proposed here, iC3b allows tumors to mask neoantigens by labeling themselves as 'super-self'. By not generating C3d, cancer cells would then preclude the cis signaling needed for IC activation. Localizing iC3b and C3d fragments within tumors using appropriate antibodies would help experimentally validate this mechanism.

The battle between iC3b and C3d is likely mediated through engagement of the cytoskeleton by CR3, analogous to those events experimentally observed during antibodymediated phagocytosis. ${ }^{10}$ The front line of the proposed clash is depicted by the ring in figure 2C. In this scenario, attachment of iC3b bound, extended CR3 $(\mathrm{E}+\mathrm{H}+)$ to actin generates forces that move proteins in the direction the fibers point. When unopposed, the strain compels the synapse to collapse. By inhibiting actin attachment within the synapse, C3d bound to bent CR3(E-H+) prevents synapse closure. Instead, the cytoskeletal forces become unbalanced and pull outwards to expand the synapse (figure 2B). Repair of the resulting membrane disruption by endosomal vesicles then allows the delivery of new antigen receptor complexes to the synapse outer edge, enhancing signaling. ${ }^{13}$

While the effects of iC3b and C3d described here are on the local IS, they also alter the makeup of cell membrane fragments released when tumor cells die. Whether the membranes bear C3d or iC3b affects the nature of the IR induced at distal sites, like lymph nodes. Killing tumor cells is not enough; they need to be tagged the right way to induce antitumor immunity.

\section{TUMORS OFTEN DELIVER A BACK-HANDED COMPLEMENT}

Tumors can influence outcomes by taking over complement production. Those that are capable of inducing immune silencing have a survival advantage. They can produce complement components internally. Alternatively, tumor cells may import $\mathrm{C} 3_{(\mathrm{H} 2 \mathrm{O})}$, which forms spontaneously in the extracellular environment when water hydrolyzes C3, along with complement factor $\mathrm{H}(\mathrm{CFH})$ and complement factor I protease. Internal cleavage of C3 then occurs, ${ }^{14}$ with the possibility that iC3b is returned to the cell surface, with CFH uptake promoting surface deposition of iC3b as it does during apoptosis. ${ }^{15}$ Intratumoral bacteria represent another possible source of complement activation, with iC3b deposition on the cancer cell membrane guaranteed by the RCAs present there.

\section{FUTURE COMPLEMENTARY DIRECTIONS}

Targeted delivery of C3d to the tumor membrane offers a new approach for inducing neoantigen-specific IRs against tumors. Amplification of the IC generated likely requires administration with other immunomodulators. Combining current agents with C3 checkpoint modulators should reduce immune adverse events, not enhance them, as immunomodulators will be more effective when dosed at levels below those that cause a breach of self-tolerance.

\section{Twitter Alan Herbert @insideoutbio}

Acknowledgements InsideOutBio acknowledges and thanks the many talented investigators whose work cannot be cited within this article format.

Contributors AH conceived, wrote, illustrated and edited the manuscript.

Funding The authors have not declared a specific grant for this research from any funding agency in the public, commercial or not-for-profit sectors.

Disclaimer No external funding was received for this work.

Competing interests The author is the founder the company Inside0utBio that is committed to open science and working across disciplines. The company is actively developing complement therapeutics for the immunotherapy of cancer. The information presented here is all derived from publicly available sources.

\section{Patient consent for publication Not required.}

Provenance and peer review Not commissioned; externally peer reviewed.

Open access This is an open access article distributed in accordance with the Creative Commons Attribution Non Commercial (CC BY-NC 4.0) license, which permits others to distribute, remix, adapt, build upon this work non-commercially, and license their derivative works on different terms, provided the original work is properly cited, appropriate credit is given, any changes made indicated, and the use is non-commercial. See http://creativecommons.org/licenses/by-nc/4.0/.

\section{ORCID iD}

Alan Herbert http://orcid.org/0000-0002-0093-1572

\section{REFERENCES}

1 Pio R, Ajona D, Ortiz-Espinosa S, et al. Complementing the CancerImmunity cycle. Front Immunol 2019;10.

2 Bajic G, Degn SE, Thiel S, et al. Complement activation, regulation, and molecular basis for complement-related diseases. Embo $J$ 2015;34:2735-57.

3 Xue X, Wu J, Ricklin D, et al. Regulator-dependent mechanisms of $\mathrm{C} 3 \mathrm{~b}$ processing by factor I allow differentiation of immune responses. Nat Struct Mol Biol 2017;24:643-51.

4 Fagerholm SC, Guenther C, Llort Asens M, et al. Beta2-Integrins and interacting proteins in leukocyte trafficking, immune suppression, and immunodeficiency disease. Front Immunol 2019;10:254.

5 Davis SJ, van der Merwe PA. The kinetic-segregation model: TCR triggering and beyond. Nat Immunol 2006;7:803-9.

6 Alcorlo M, López-Perrote A, Delgado S, et al. Structural insights on complement activation. Febs J 2015;282:3883-91.

7 Fan Z, Kiosses WB, Sun H, et al. High-Affinity bent $\beta 2$-Integrin molecules in arresting neutrophils face each other through binding to ICAMs in cis. Cell Rep 2019;26:119-30.

8 Adair BD, Xiong J-P, Alonso JL, et al. EM structure of the ectodomain of integrin CD11b/CD18 and localization of its ligand-binding site relative to the plasma membrane. PLoS One 2013;8:e57951.

9 Varga G, Balkow S, Wild MK, et al. Active MAC-1 (CD11b/CD18) on DCs inhibits full T-cell activation. Blood 2007;109:661-9.

10 Freeman SA, Goyette J, Furuya W, et al. Integrins form an expanding diffusional barrier that coordinates phagocytosis. Cell 2016:164:128-40.

11 Borschukova O, Paz Z, Ghiran IC, et al. Complement fragment C3d is colocalized within the lipid rafts of T cells and promotes cytokine production. Lupus 2012;21:1294-304.

12 Platt JL, Silva I, Balin SJ, et al. C3d regulates immune checkpoint blockade and enhances antitumor immunity. JCl Insight 2017;2.

13 Onnis A, Baldari CT. Orchestration of immunological synapse assembly by vesicular trafficking. Front Cell Dev Biol 2019;7:110.

14 Elvington M, Liszewski MK, Bertram P, et al. A C3(H20) recycling pathway is a component of the intracellular complement system. $J$ Clin Invest 2017;127:970-81.

15 Martin M, Leffler J, Smoląg Kl, et al. Factor $\mathrm{H}$ uptake regulates intracellular $\mathrm{C} 3$ activation during apoptosis and decreases the inflammatory potential of nucleosomes. Cell Death Differ 2016;23:903-11.

16 Huber-Lang M, Ekdahl KN, Wiegner R, et al. Auxiliary activation of the complement system and its importance for the pathophysiology of clinical conditions. Semin Immunopathol 2018;40:87-102.

17 Sen M, Yuki K, Springer TA, et al. An internal ligand-bound, metastable state of a leukocyte integrin, $\alpha \times \beta 2$. J Cell Biol 2013;203:629-42. 